Sanz-Arribas, I.; Martínez de Haro, V.; Calle-Molina, M.T. (2020) Evolution of the Competence in Water of the Aquatic Lifeguards. Revista Internacional de Medicina y Ciencias de la Actividad $\begin{array}{lllllll}\text { Física y el } & \text { Deporte } & \text { vol. } 20 & (80) & \text { pp. } & 595-609\end{array}$ Http://cdeporte.rediris.es/revista/revista80/artevolucion1179.htm

DOI: https://doi.org/10.15366/rimcafd2020.80.009

\title{
ORIGINAL
}

\section{EVOLUCIÓN DE LA COMPETENCIA EN EL AGUA DE LOS SOCORRISTAS ACUÁTICOS}

\section{EVOLUTION OF THE COMPETENCE IN WATER OF THE AQUATIC LIFEGUARDS}

\author{
Sanz-Arribas, I.; Martínez-de-Haro, V. y Calle-Molina, M.T. \\ Departamento de Educación Física, Deporte y Motricidad Humana. Universidad Autónoma de \\ Madrid (España) ismael.sanz@uam.es; vicente.martinez@uam.es; mariat.calle@uam.es
}

\section{Agradecimientos}

Todos los datos que han permitido la elaboración de este estudio han sido facilitados por la Federación Madrileña de Salvamento y Socorrismo, por lo tanto, en este apartado se agradece la inestimable colaboración de esta entidad y de su equipo de profesionales.

Código UNESCO / UNESCO CODE: 2411.99 Otras (Actividad Física y Salud) / Others (Physical Activity and Health)

Clasificación del Consejo de Europa / European Council Classification: 17 Otras (Actividad Física y Salud) / Others (Physical Activity and Health)

Recibido 19 de noviembre de 2018 Received November 19, 2018 Aceptado 7 de septiembre de 2019 Accepted September 7, 2019

\section{RESUMEN}

En el año 2006 entró en vigor la normativa que actualmente regula la formación de socorristas en la Comunidad Autónoma de Madrid (España). El objetivo de este estudio es conocer la influencia de la aplicación de esta normativa sobre el porcentaje de aspirantes que obtiene el diploma de socorrista acuático y sobre su nivel de competencia en el agua. Para ello, se han analizado las marcas de tiempo acreditadas por 6.105 aspirantes a socorrista (4.288 hombres y 1.817 mujeres) que se formaron entre el año 1993 y 2016. Los resultados demuestran que, desde la entrada en vigor de dicha normativa, se ha incrementado el porcentaje de aspirantes que obtiene el diploma de socorrista acuático, pero se ha disminuido su nivel de competencia en el agua. Se recomienda que las instituciones competentes establezcan objetivos y criterios de evaluación que promuevan la mejora del nivel de competencia en el agua de estos profesionales. 
PALABRAS CLAVE: socorrista acuático, salvamento y socorrismo, ahogamiento, condición física.

\begin{abstract}
In 2006, the normative that currently regulates the training of lifeguards in the Autonomous Community of Madrid (Spain) came into force. The objective of this study is to know the influence of the application of this regulation on the percentage of applicants who obtain the aquatic lifeguard diploma and on their level of competence in the water. For this purpose, the time marks, accredited by 6,105 applicants to lifeguards (4,288 men and 1,817 women) who received this training between 1993 and 2016, have been analysed. The results show that, since the regulation came into force, the percentage of applicants who obtain the lifeguard diploma has increased, but their level of competence in the water has decreased. Therefore, it is recommended that objectives and assessment criteria that are established by the institutions, encourage the improvement of the level of competence in the water of these professionals.
\end{abstract}

KEYWORDS: lifeguard, lifesaving, rescue, drowning, physical condition.

\title{
INTRODUCCIÓN
}

Está demostrado que la presencia de socorristas en los espacios acuáticos es una de las mejores formas de prevenir el ahogamiento y la muerte accidental en el agua (Harrell, 2001; Pelletier \& Gilchrist, 2011; Schwebel, Heater, Holder, \& Marciani, 2010). Es más, se ha podido constatar que las instalaciones acuáticas que no están vigiladas por socorristas sufren una mayor prevalencia de víctimas mortales en el agua, que aquellas que sí que lo están. En el caso concreto de España, el 84,9\% de las 2.589 personas que murieron accidentalmente en el agua desde el año 2015 hasta el año 2019, lo hicieron en un espacio acuático en el que no existía servicio de socorrismo o en el que no había socorristas en el momento de producirse el accidente (Real Federación Española de Salvamento y Socorrismo, 2019). Por lo tanto, es necesario que las entidades competentes contraten socorristas en aquellos espacios acuáticos que la población utiliza habitualmente. También es preciso convencer a la población para que sólo haga uso de los entornos acuáticos vigilados por estos profesionales. Sin embargo, los resultados de este informe también advierten que, a lo largo de este mismo periodo, un total de 384 personas fallecieron en aguas vigiladas por socorristas. Este dato confirma que aún existe un importante margen de mejora en la seguridad de los espacios acuáticos vigilados en España. A este respecto, la bibliografía establece que los socorristas deben conocer y aplicar las medidas de prevención y vigilancia adecuadas al entorno de baño en el que ejercen. También se considera imprescindible que este personal sanitario domine los primeros auxilios y que disponga de un alto nivel de competencia en el agua para poder realizar rescates acuáticos con éxito (Consejería de Sanidad y Servicios Sociales de la Comunidad de Madrid, 1998; Ellis \& Associates, 2002; García Sanz, García Sanz, \& Díez Herrero, 2015; 
International Life Saving Federation, 2000, 2007, 2013; Palacios Aguilar, 2008; Sanz Arribas, 2011; The United States Lifesaving Association, 2016).

Aunque todos estos factores condicionan la calidad del servicio de socorrismo, este estudio se centra específicamente en la importancia del nivel de competencia en el agua del socorrista acuático y su dominio de las habilidades específicas del salvamento y socorrismo. En relación con esta cuestión, es evidente que realizar rescates acuáticos en el menor tiempo posible, incrementa las posibilidades de supervivencia de la persona rescatada y disminuye las secuelas generadas por la hipoxia que habitualmente sufren las víctimas ahogadas (Austin \& Macintosh, 2013; Martínez \& Hooper, 2014). También está demostrado que el retraso en el inicio de las maniobras de resucitación, influye negativamente en el resultado final del salvamento (Neumar et al., 2015; Szpilman et al., 2014). De hecho, algunos autores consideran que siempre que sea posible y que no comprometa la seguridad de los socorristas, merece la pena iniciar las maniobras de resucitación en los rescates acuáticos, incluso antes de que la víctima sea trasladada hasta la orilla, aunque sobre este asunto existe controversia por la dificultad de realizar un soporte vital básico adecuado antes de la llegada a tierra firme (Szpilman \& Marcio, 2004). Al margen del tiempo de rescate acuático, también se ha demostrado que cuando el nivel de fatiga de la persona que realiza las maniobras de resucitación es elevado, la efectividad de dichas maniobras disminuye significativamente y por lo tanto, las posibilidades de éxito del salvamento se desvanecen (Abelairas Gómez, Romo Pérez, \& Barcala Furelos, 2013). Las conclusiones de todos estos estudios hacen pensar que disponer de un mayor nivel de competencia en el agua, incrementa las posibilidades de éxito del salvamento, por la reducción del tiempo de rescate acuático, por el adelanto en el inicio de las maniobras de resucitación y por la disminución del estado de fatiga del rescatador.

Con independencia de lo anterior, valorar el nivel de competencia en el agua del socorrista es una cuestión de gran importancia, por el considerable riesgo que asume este profesional en los rescates acuáticos. Concretamente, desde enero de 2015 hasta diciembre de 2019, en España han fallecido 45 personas que intentaron rescatar del agua a otras personas o incluso a sus mascotas (Real Federación Española de Salvamento y Socorrismo, 2019). Estas cifras muestran claramente el enorme riesgo que asume una persona que trata de realizar un rescate acuático, especialmente si el rescatador no está debidamente preparado.

Por estas razones, la mayoría de las entidades y organismos públicos y privados que regulan la formación de socorristas acuáticos, establecen que los aspirantes a esta certificación superen criterios de evaluación vinculados al nivel de competencia en el agua. Desafortunadamente y a pesar de las evidencias presentadas anteriormente, se han encontrado diferencias importantes entre los criterios de evaluación fijados por diversas instituciones y/o normas que regulan la formación de socorristas acuáticos (Consejería de Sanidad y Consumo, 2006; Cruz Roja, 2020; Federación Madrileña de Salvamento y Socorrismo, 2017; International Life Saving Federation, 2000, 2013; Ministerio de Trabajo e Inmigración, 2011; The United States Lifesaving Association, 2016). Precisamente, la falta crónica de estandarización en el nivel de exigencia de las 
pruebas físicas de agua, podría estar provocando que el grado de competencia en el agua de los socorristas acuáticos capacitados para trabajar en entornos similares no sea homogéneo. Consecuentemente, es necesario regular y homogeneizar la formación y los criterios de evaluación de esta profesión. Un ejemplo de homogeneización de los criterios de evaluación es el que ha supuesto la aplicación de la normativa vigente en la Comunidad Autónoma de Madrid desde el año 2006 (Consejería de Sanidad y Consumo, 2006). Antes de la aplicación de la mencionada normativa, no existía un criterio unificado para formar a los socorristas acuáticos en este territorio. En consecuencia, las diferentes entidades formadoras de socorristas acuáticos decidían unilateralmente los objetivos, los contenidos, los criterios de evaluación y la carga lectiva de sus cursos, con el evidente riesgo de heterogeneidad formativa que esta circunstancia podría generar.

No obstante, además de estandarizar la formación, también es preciso que el nivel de exigencia sea lo suficientemente alto como para que la seguridad de los bañistas y de los socorristas no se vea comprometida. En este sentido, se ha podido constatar que dicha normativa (Consejería de Sanidad y Consumo, 2006), plantea unos criterios de evaluación diferentes a los que se venían aplicando por parte de algunas de las entidades con más experiencia en la formación de socorristas acuáticos. En concreto y antes de la aplicación de dicha normativa, la Federación Madrileña de Salvamento y Socorrismo (FMSS) que es la entidad que ha cedido los datos para la elaboración de este estudio, establecía que los aspirantes a socorrista acuático debían superar todas y cada una de las diez pruebas de piscina (nueve pruebas cronometradas y una apnea). En cambio, y con independencia de la entidad formadora en la que los aspirantes decidan formarse, desde la entrada en vigor de la citada orden, todas las personas que actualmente aspiran a este diploma en la Comunidad Autónoma de Madrid deben superar tres pruebas físicas acuáticas para poder ejercer como socorrista en una piscina, y una cuarta prueba más, para aquellos que quieren ejercer esta profesión en espacios acuáticos naturales. En resumen, uno de los criterios de evaluación que no han sido modificados por la citada orden, es la necesidad de superar todas y cada una de las pruebas físicas de agua vigentes para obtener el diploma.

A continuación, se muestra una tabla en la que se detalla lo descrito en este párrafo:

Tabla 1: Relación de pruebas aplicadas por la entidad que ha cedido los datos para este estudio (FMSS) antes de la aplicación de la Orden 1319/2006 y relación de pruebas

establecidas en la Comunidad de Madrid, desde la aplicación de la citada norma:

\begin{tabular}{|c|c|}
\hline $\begin{array}{l}\text { Pruebas físicas de agua exigidas por } \\
\text { la FMSS, antes de la aplicación de la } \\
\text { norma de } 2006\end{array}$ & $\begin{array}{c}\text { Pruebas físicas de agua exigidas en la } \\
\text { Comunidad Autónoma de Madrid, } \\
\text { después la aplicación de la norma de } \\
2006\end{array}$ \\
\hline $\begin{array}{l}\text { 1. } 200 \text { metros libres (tiempo máximo } 4 \\
\text { minutos y } 15 \text { segundos). } \\
\text { 2. } 100 \text { metros libres (tiempo máximo } 1 \\
\text { minuto y } 40 \text { segundos). } \\
\text { 3. } 300 \text { metros libres con ropa (tiempo } \\
\text { máximo } 8 \text { minutos). }\end{array}$ & $\begin{array}{l}\text { 1. } 300 \text { metros libres (tiempo máximo } 8 \\
\text { minutos). } \\
\text { 2. } 100 \text { metros combinada de salvamento } \\
\text { I con material de rescate (tiempo } \\
\text { límite } 3 \text { minutos y } 30 \text { segundos). }\end{array}$ \\
\hline
\end{tabular}




\begin{tabular}{|c|c|}
\hline $\begin{array}{l}\text { 4. } 300 \text { metros libres con aletas (tiempo } \\
\text { máximo } 6 \text { minutos). } \\
\text { 5. } 100 \text { metros combinada I (tiempo } \\
\text { máximo } 3 \text { minutos } 30 \text { segundos). } \\
\text { 6. } 100 \text { metros combinada de } \\
\text { salvamento II (tiempo máximo } 3 \\
\text { minutos). } \\
\text { 7. } 100 \text { metros remolque de accidentado } \\
\text { (tiempo límite } 4 \text { minutos). } \\
\text { 8. } 100 \text { metros remolque de accidentado } \\
\text { con aletas (tiempo límite } 3 \text { minutos y } \\
30 \text { segundos). } \\
\text { 9. } 50 \text { metros rescate de accidentado con } \\
\text { material (tiempo límite } 1 \text { minuto } 45 \\
\text { segundos). } \\
\text { 10. } 25 \text { metros de buceo (sin límite de } \\
\text { tiempo). }\end{array}$ & $\begin{array}{l}\text { 3. } \mathbf{1 0 0} \text { metros combinada de } \\
\text { salvamento II (tiempo máximo } \mathbf{3} \\
\text { minutos). } \\
\text { 4. } 100 \text { metros rescate de accidentado } \\
\text { con aletas ( } 50 \text { metros nado de } \\
\text { aproximación y } 50 \text { metros traslado de } \\
\text { accidentado en un tiempo máximo } 3 \\
\text { minutos } 30 \text { segundos). Esta prueba } \\
\text { es diferente a la prueba de } 100 \\
\text { metros remolque de accidentado con } \\
\text { aletas. Además, sólo debe ser } \\
\text { superada por aquellos aspirantes que } \\
\text { deseen obtener el diploma de } \\
\text { socorrista en espacios acuáticos } \\
\text { naturales. }\end{array}$ \\
\hline
\end{tabular}

Nota 1: Como puede observarse en la tabla presentada, la única prueba que la entidad que ha cedido los datos ha estado utilizando antes y después de la aplicación de la citada norma, es la prueba "100 metros combinada de salvamento II" marcada en negrita en la tabla 1 . Por lo tanto,

las marcas de tiempo acreditadas antes de la norma de 2006, pueden compararse con las obtenidas después de la aplicación de esta norma.

\section{OBJETIVO}

El objetivo de este estudio es conocer la influencia de la aplicación de esta norma sobre el porcentaje de aspirantes que obtiene el diploma de socorrista acuático y sobre su nivel de competencia en el agua.

\section{METODOLOGÍA}

\section{Normas éticas}

Los datos que han permitido la elaboración de este estudio han sido cedidos por la Federación Madrileña de Salvamento y Socorrismo (FMSS). Para la cesión de estos datos se ha garantizado que toda la información es tratada con una finalidad exclusivamente investigadora. A excepción del sexo de las personas que fueron evaluadas por esta institución, no se han incluido datos personales 0 sensibles que permitiesen la identificación de las personas implicadas en esta investigación y, por lo tanto, su anonimato queda totalmente garantizado. En definitiva, se han respetado todas las normas éticas de investigación, tanto nacionales como internacionales y en ningún caso se han invadido los límites de privacidad y respeto hacia las personas.

\section{Características de la muestra}


En este estudio se han analizado las marcas de tiempo obtenidas por 6.105 aspirantes al diploma de socorrista acuático (4.288 hombres y 1.817 mujeres). Las marcas analizadas se corresponden con el tiempo acreditado por dichos aspirantes al realizar la prueba denominada "100 metros combinada de salvamento II". Dichas marcas fueron cronometradas por el equipo docente de la Federación Madrileña de Salvamento y Socorrismo y posteriormente archivadas por el personal administrativo de esta entidad. El período de tiempo que abarca esta investigación comprende desde el año 1993 hasta el año 2016.

Todas las personas que realizaron la citada prueba debían cumplir con los siguientes requisitos para poder acceder al curso de formación de socorrista acuático:

- Ser mayores de 16 años y haber superado el $4^{\circ}$ curso de la Enseñanza Secundaria Obligatoria (E.S.O.) o equivalente (Plan de estudios de España).

- Presentar un certificado médico oficial, en el que constase que no padecían alguna enfermedad infectocontagiosa y que eran aptos para realizar ejercicio físico en el agua.

- Las personas menores de 18 años debían presentar una autorización firmada por sus padres o tutores legales, en la que se les autorizaba a realizar las actividades necesarias para la obtención del diploma de socorrista acuático.

\section{Material y métodos}

\section{Recursos humanos y materiales}

- Equipo docente de la Federación Madrileña de Salvamento y Socorrismo. Este equipo docente reúne los requisitos necesarios para poder formar socorristas en la Comunidad Autónoma de Madrid.

- Personal administrativo de la Federación Madrileña de Salvamento y Socorrismo.

- Instalaciones acuáticas con vasos de 50 y 25 metros de longitud y con 180 centímetros de profundidad mínima en el lugar de colocación del maniquí.

- Maniquíes de salvamento y socorrismo acuático utilizados por la Real Federación Española de Salvamento y Socorrismo. Las pruebas que requieren de la utilización de este material siempre se realizaron con el mismo modelo de maniquí. Este material se caracteriza por ser estanco y por estar completamente lleno de agua en el momento de la realización de las pruebas. 
- Cronómetros del modelo Casio HS-30W, resistentes al agua y con memoria para 10 parciales.

- Silbatos para dar la señal de comienzo de la prueba cronometrada.

- Todos los participantes del estudio realizaron las pruebas sin gafas de natación.

\section{Descripción y criterios de selección de la prueba}

A continuación, se describe la prueba "100 metros combinada de salvamento II": entrada al agua con avistamiento de la víctima. El aspirante debe nadar 50 metros de aproximación y 15 metros de buceo ininterrumpido hasta la recogida de una víctima (maniquí) que se encuentra en el fondo del vaso. Por último, el aspirante debe realizar 35 metros de traslado del maniquí, sin que las vías respiratorias de éste se vean obstaculizadas por el agua o por las manos del aspirante. El tiempo límite de esta prueba es de 3 minutos. Se destaca que en esta prueba siempre se utiliza un maniquí normalizado, lleno de agua y estanco, que ha sido descrito en el apartado de recursos materiales. Esto significa que la "víctima" está estandarizada y es inerte. Por otro lado, la normativa de Comunidad Autónoma de Madrid establece que la profundidad mínima a la que se debe encontrar el maniquí durante la prueba es de 180 centímetros y que la longitud mínima del vaso en el que se desarrollan las pruebas de agua debe ser de 25 metros (Consejería de Sanidad y Consumo, 2006). Esto último significa que las pruebas cronometradas de agua pueden evaluarse en vasos de veinticinco metros de longitud o de cincuenta. Puesto que la información cedida por la institución ha permitido conocer la longitud del vaso en el que se evaluó a la muestra, se han separado las marcas de tiempo obtenidas en vasos de veinticinco metros, de aquellas que fueron obtenidas en vasos de cincuenta metros. Esta decisión se debe a que la longitud del vaso en el que se evalúan estas pruebas, influye significativamente sobre las marcas que acreditan los aspirantes a socorrista (Sanz-Arribas, 2018).

La elección de este test se debe a que es una prueba física que está ampliamente recomendada para la evaluación del nivel de competencia en el agua del socorrista y de su capacidad para desempeñar un rescate acuático con éxito (Consejería de Sanidad y Consumo, 2006; Cruz Roja, 2020; Federación Madrileña de Salvamento y Socorrismo, 2017). Asimismo, es una prueba física muy similar a la que se desarrolla en las competiciones de la modalidad deportiva de salvamento y socorrismo (International Life Saving Federation, 2019), disciplina deportiva que, entre otras cosas, se caracteriza por valorar el tiempo empleado para desempeñar diferentes rescates acuáticos simulados. Además, y tal y como se ha mencionado con anterioridad, esta es la única prueba que está incluida en la batería de pruebas que establece la normativa citada y que, al mismo tiempo, también estaba incluida entre las pruebas que aplicaba la entidad que ha cedido los datos de este estudio. Esta última circunstancia, permite comparar las marcas de tiempo acreditadas por los aspirantes que obtienen el diploma de socorrista en una misma prueba, antes y después de la aplicación de la citada norma. 


\section{Desarrollo del estudio}

Para conocer la influencia de la aplicación de la norma sobre el nivel de competencia en el agua de los aspirantes a socorrista acuático, se han comparado las marcas de tiempo obtenidas por los aspirantes que fueron evaluados antes de su aplicación, con las marcas obtenidas por los aspirantes que fueron evaluados después de la entrada en vigor de la citada norma.

En relación con el porcentaje de aspirantes que obtiene el diploma de socorrista acuático, se ha tenido en cuenta que, para la obtención de dicho diploma, es necesario superar todas y cada de las pruebas mostradas en la tabla 1. Por lo tanto, aquellos aspirantes que no superaron alguna de las pruebas propuestas antes o después de la aplicación de la norma, se han considerado como no aptos.

Por último, se aclara que se ha decidido no valorar la influencia del sexo sobre los objetivos planteados para este estudio, porque se considera que las personas que hacen uso de los espacios acuáticos vigilados por socorristas, únicamente están interesadas en conocer que el o la socorrista es competente para ejercer esta profesión y no en su sexo. No obstante, se indica que el porcentaje de mujeres y de hombres que fue evaluado en este estudio es muy similar en los dos periodos de tiempo analizados. Concretamente, entre 1993 y 2006, se evaluó a un $29,8 \%$ de mujeres y a un $70,2 \%$ de hombres. Respecto al periodo comprendido en 2006 y 2016 , se evaluó a un $28,9 \%$ de mujeres y a un $71,1 \%$ de hombres.

\section{RESULTADOS}

Gráfico 1: Porcentaje de aspirantes a socorrista acuático, aptos y no aptos, antes de la aplicación de la norma

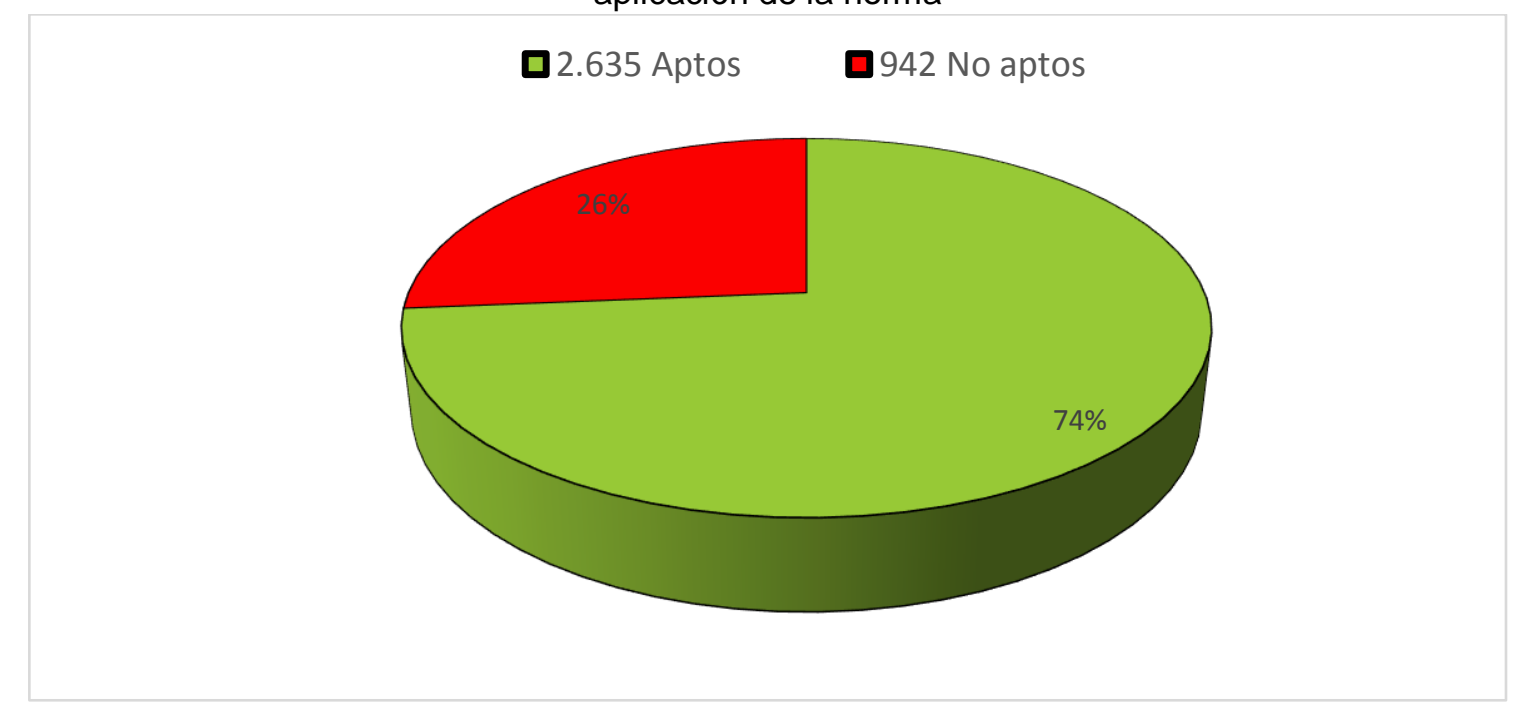

Gráfico 2: Porcentaje de aspirantes a socorrista acuático, aptos y no aptos, después de la aplicación de la norma 


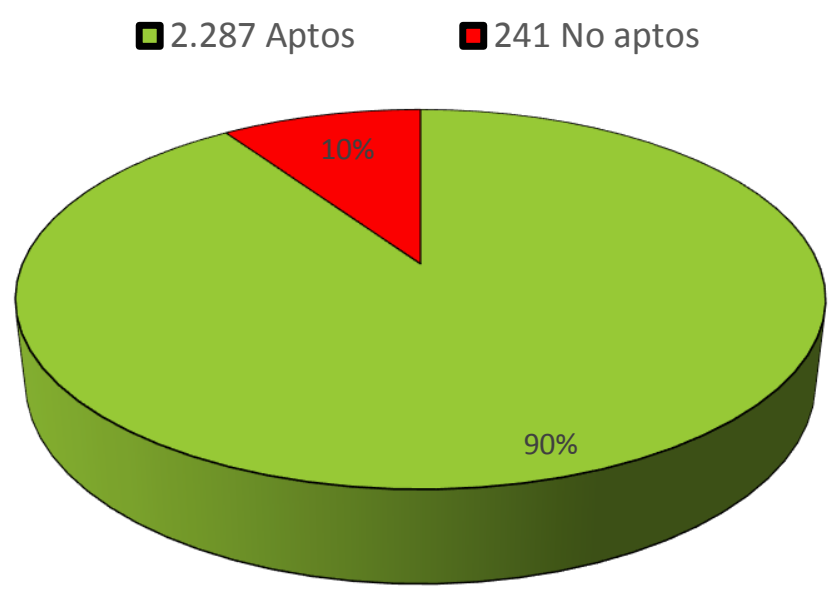

Tabla 2: Evolución de las marcas de tiempo desde el año 1993 hasta el año 2016, en la prueba "100 metros combinada de salvamento II" obtenidas en vasos de 50 metros

\begin{tabular}{|c|c|c|c|c|c|}
\hline \multicolumn{7}{|c|}{ Estadísticos de grupo } \\
\hline & $\begin{array}{c}\text { Anteriores } \\
\text { posteriores a la Norma } \\
\text { de 2006 }\end{array}$ & $\mathrm{N}$ & Media & $\begin{array}{c}\text { Desviación } \\
\text { típ. }\end{array}$ & $\begin{array}{c}\text { Error típ. De } \\
\text { la media }\end{array}$ \\
\hline $\begin{array}{c}\text { Tiempo de la } \\
\text { combinada II en } \\
\text { segundos }\end{array}$ & $\begin{array}{c}\text { Desde 1993 hasta } \\
2006\end{array}$ & 1.486 & 135,57 & 15,63 &, 405 \\
\cline { 2 - 6 } & $\begin{array}{c}\text { Desde 2006 hasta } \\
2016\end{array}$ & 565 & 153,21 & 19,23 &, 809 \\
\hline
\end{tabular}

Gráfico 3: Evolución de las marcas de tiempo en la prueba "100 metros combinada de salvamento II" obtenidos en vasos de 50 metros

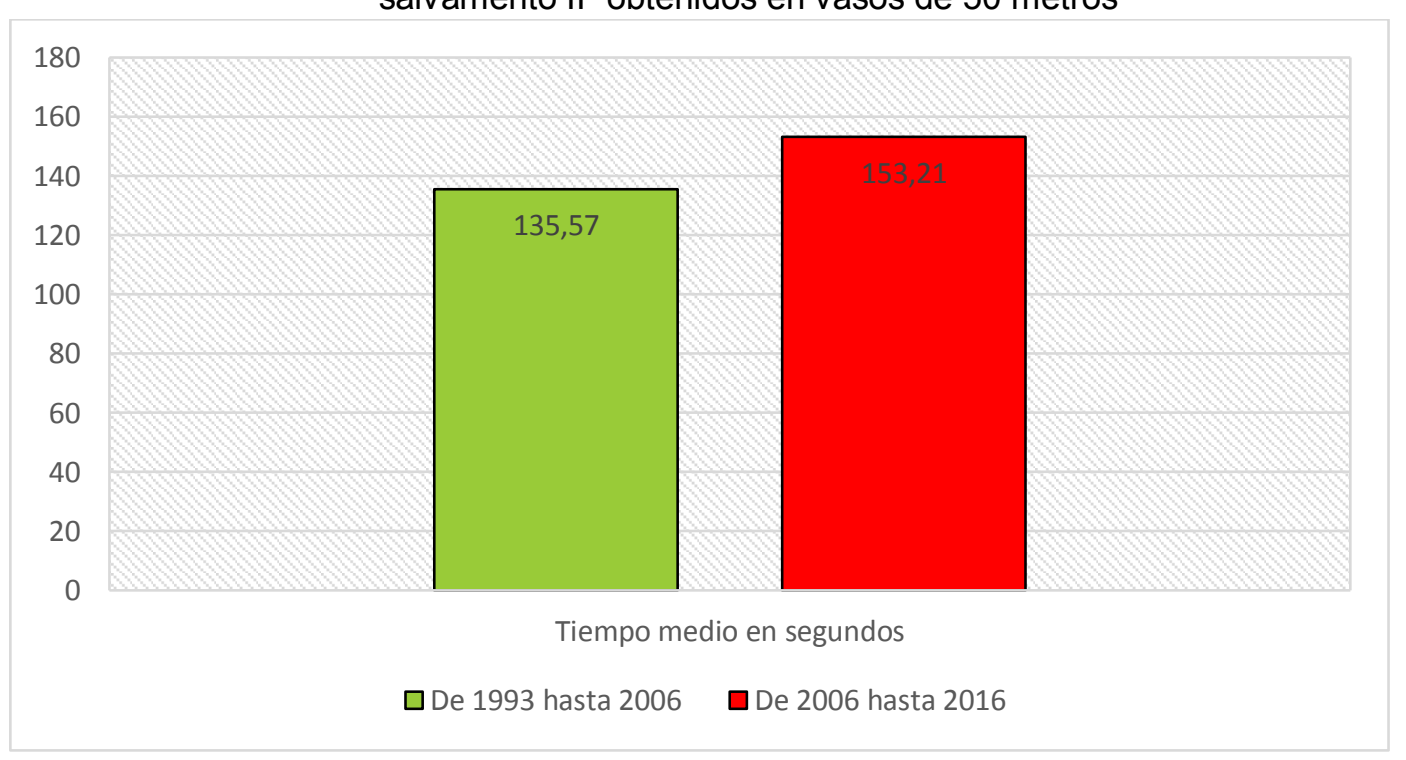

Tabla 3: Resultados de la prueba t en vasos de 50 metros 


\begin{tabular}{|c|c|c|c|c|c|c|c|c|c|}
\hline \multicolumn{10}{|c|}{ Prueba de muestras independientes } \\
\hline \multirow{4}{*}{$\begin{array}{l}\text { Evolución de } \\
\text { las marcas de } \\
\text { tiempo en la } \\
\text { prueba } 100 \\
\text { metros } \\
\text { combinada II. } \\
\text { (Vaso de } 50 \\
\text { metros) }\end{array}$} & \multicolumn{2}{|c|}{\begin{tabular}{|lr} 
Prueba & de \\
Levene para la \\
igualdad & de \\
varianzas & \\
\end{tabular}} & \multicolumn{7}{|c|}{ Prueba t para la igualdad de medias } \\
\hline & \multirow[b]{2}{*}{$\mathrm{F}$} & \multirow[b]{2}{*}{ Sig. } & \multirow[b]{2}{*}{$\mathrm{T}$} & \multirow[b]{2}{*}{ gl } & \multirow{2}{*}{$\begin{array}{c}\text { Sig. } \\
\text { (bilateral } \\
\text { ) }\end{array}$} & \multirow{2}{*}{$\begin{array}{l}\text { Diferenc } \\
\text { ia de } \\
\text { medias }\end{array}$} & \multirow[b]{2}{*}{$\begin{array}{l}\text { Error típico de } \\
\text { la diferencia }\end{array}$} & \multicolumn{2}{|c|}{$\begin{array}{c}\text { 95\% Intervalo } \\
\text { de confianza } \\
\text { para la } \\
\text { diferencia }\end{array}$} \\
\hline & & & & & & & & $\begin{array}{c}\text { Inferio } \\
r\end{array}$ & $\begin{array}{l}\text { Superi } \\
\text { or }\end{array}$ \\
\hline & $\begin{array}{c}46,5 \\
77\end{array}$ & ,000 & $\begin{array}{c}- \\
21,372\end{array}$ & 2049 & ,000 & $-17,64$ & ,82 & $-19,25$ & $-16,02$ \\
\hline
\end{tabular}

Tabla 4: Evolución de las marcas de tiempo desde el año 1996, hasta el año 2016, en la prueba "100 metros combinada de salvamento II" obtenidas en vasos de 25 metros

\begin{tabular}{|c|c|c|c|c|c|}
\hline \multicolumn{7}{|c|}{ Estadísticos de grupo } \\
\hline & $\begin{array}{c}\text { Anteriores y } \\
\text { posteriores a la Norma } \\
\text { de 2006 }\end{array}$ & $\mathrm{N}$ & Media & $\begin{array}{c}\text { Desviación } \\
\text { típ. }\end{array}$ & $\begin{array}{c}\text { Error típ. } \\
\text { De la media }\end{array}$ \\
\hline $\begin{array}{c}\text { Tiempo de la } \\
\text { combinada II en } \\
\text { segundos }\end{array}$ & $\begin{array}{c}\text { Desde 1993 hasta } \\
2006\end{array}$ & 879 & 138,16 & 16,649 &, 562 \\
\cline { 2 - 6 } & $\begin{array}{c}\text { Desde 2006 hasta } \\
2016\end{array}$ & 1.719 & 148,86 & 19,434 &, 469 \\
\hline
\end{tabular}

Gráfico 4: Evolución de las marcas de tiempo en la prueba "100 metros combinada de salvamento II" obtenidos en vasos de 25 metros.

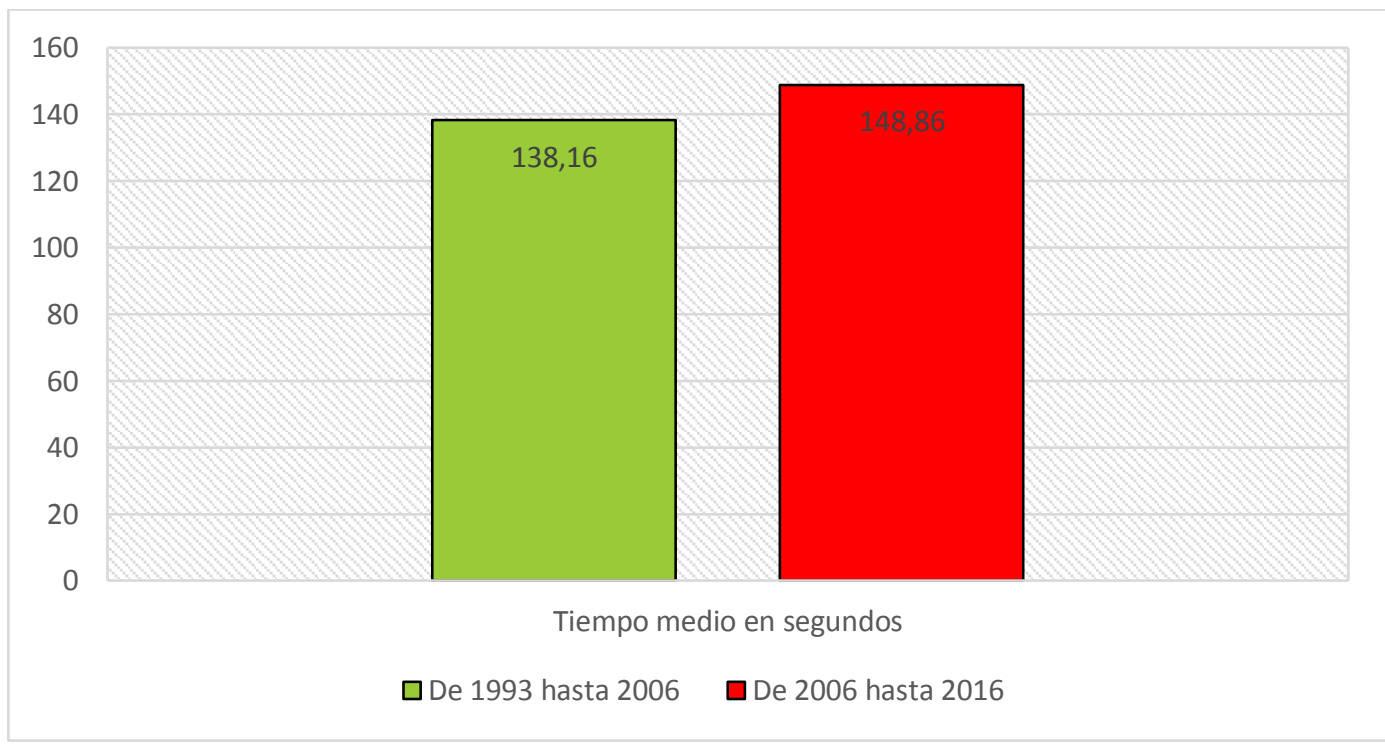

Tabla 4: Resultados de la prueba t en vasos de 25 metros. 


\begin{tabular}{|c|c|c|c|c|c|c|c|c|c|}
\hline \multicolumn{10}{|c|}{ Prueba de muestras independientes } \\
\hline \multirow{4}{*}{$\begin{array}{c}\text { Evolución de } \\
\text { las marcas } \\
\text { de tiempo en } \\
\text { la prueba } \\
100 \text { metros } \\
\text { combinada } \\
\text { II. } \\
\text { (Vaso de } 25 \\
\text { metros) }\end{array}$} & \multicolumn{2}{|c|}{$\begin{array}{l}\text { Prueba de } \\
\text { Levene para } \\
\text { la igualdad } \\
\text { de varianzas }\end{array}$} & \multicolumn{7}{|c|}{ Prueba t para la igualdad de medias } \\
\hline & \multirow[b]{2}{*}{$F$} & \multirow[b]{2}{*}{ Sig. } & \multirow[b]{2}{*}{$\mathrm{T}$} & \multirow[b]{2}{*}{ gl } & \multirow{2}{*}{$\begin{array}{c}\text { Sig. } \\
\text { (bilateral } \\
\text { ) }\end{array}$} & \multirow{2}{*}{$\begin{array}{c}\text { Diferenc } \\
\text { ia de } \\
\text { medias }\end{array}$} & \multirow[b]{2}{*}{$\begin{array}{l}\text { Error típico de } \\
\text { la diferencia }\end{array}$} & \multicolumn{2}{|c|}{$\begin{array}{c}\text { 95\% Intervalo } \\
\text { de confianza } \\
\text { para la } \\
\text { diferencia }\end{array}$} \\
\hline & & & & & & & & Inferior & $\begin{array}{c}\text { Superi } \\
\text { or }\end{array}$ \\
\hline & $\begin{array}{c}35,76 \\
9\end{array}$ & ,000 & $\begin{array}{c}- \\
13,916\end{array}$ & $\begin{array}{c}259 \\
6\end{array}$ & ,000 & $-10,69$ & ,769 & $-12,2$ & $-9,19$ \\
\hline
\end{tabular}

\section{DISCUSIÓN}

Antes de discutir los resultados de este trabajo, se adelanta que, todas las reflexiones y conclusiones mostradas en este documento se refieren exclusivamente a la población que ha formado parte de esta investigación. Por lo tanto, en ningún caso se pretende extrapolar los resultados de este trabajo al universo de socorristas. No obstante, debido a las características de la muestra y a la temática tratada, se recomienda que estos resultados sean tenidos en consideración a la hora de tomar decisiones que afecten a la formación de socorristas.

Los resultados de esta investigación muestran que después de la aplicación de la norma de la Comunidad Autónoma de Madrid, se ha incrementado el porcentaje de aspirantes que obtiene el diploma de socorrista acuático, pero se ha disminuido significativamente el nivel de competencia en el agua de estos profesionales. En concreto, antes de la aplicación de la norma, el 74\% de los aspirantes superaba todas las pruebas de agua, en cambio, después de su aplicación, el porcentaje de aprobados ha ascendido al $90 \%$. Con respecto al nivel de competencia en el agua de los socorristas, se puede afirmar que las marcas obtenidas en la prueba "100 metros combinada de salvamento II" antes de la aplicación de la norma de 2006, son significativamente mejores que las que se obtuvieron después de la referida norma. Este hecho se ha demostrado tanto en vasos de 25 metros, donde el tiempo medio se ha incrementado 10,7 segundos $(p \leq 0,001)$, como en vasos de 50 metros, donde el tiempo medio se ha incrementado en 17,6 segundos ( $p \leq 0,001)$. Las diferencias encontradas entre las marcas de tiempo obtenidas en los vasos de 25 metros de longitud y los de 50 metros, son compatibles con los resultados descritos en un estudio previo (Sanz-Arribas, 2018). Por lo tanto, se vuelve a poner de manifiesto la necesidad de establecer límites de tiempo diferentes, en función de la longitud del vaso en el que se desarrollen las pruebas de agua que se utilizan para evaluar a socorristas acuáticos.

Todo parece indicar que los resultados de este estudio se deben a la disminución del número y exigencia de las pruebas físicas cronometradas en el agua. Tal y como puede apreciarse en la tabla 1, antes de la aplicación de la norma, la entidad que cedió los datos exigía superar las nueve pruebas cronometradas de 
agua y una apnea. En cambio, después de la aplicación de la citada norma, en este territorio sólo se exige superar tres pruebas físicas cronometradas de agua para obtener el diploma de socorrista acuático de piscinas e instalaciones acuáticas y en el caso de que el aspirante quiera obtener el diploma de socorrista de espacios acuáticos naturales, es necesario superar una cuarta prueba más (ver tabla 1).

La disminución del número de pruebas físicas cronometradas puede contribuir a la reducción del tiempo empleado para la evaluación. Esto significa, que hay más tiempo disponible para la formación y para la práctica de las habilidades y destrezas del salvamento acuático. Consecuentemente, esta medida podría considerarse acertada, siempre que las pruebas elegidas y los límites de tiempo establecidos para ejecutarlas, sean los adecuados para valorar si el aspirante es capaz de realizar un rescate acuático con posibilidades reales de éxito. Sobre este asunto, se recuerda que la bibliografía recomienda que el socorrista sea competente en el agua y que domine las técnicas del rescate acuático con la finalidad de reducir el tiempo de rescate en el agua, minimizar el tiempo que la víctima se expone a la hipoxia y permitir que se inicien las maniobras de resucitación con posibilidades reales de éxito (Austin \& Macintosh, 2013; Martínez y Hooper, 2014; Szpilman et al., 2014; Neumar et al., 2015). A este respecto cabe decir que, según la información mostrada en la tabla 1 , parece evidente que a excepción de la prueba "100 metros combinada de salvamento II", que no ha sido modificada en modo alguno, el resto de las pruebas exigidas actualmente en este territorio, ofrecen al aspirante un amplio margen de tiempo para su superación (Sanz-Arribas, 2018) y, en cualquier caso, aparentan ser menos exigentes que las que se planteaban con anterioridad. Esta probable disminución del nivel de exigencia podría provocar que aspirantes con un menor nivel de competencia en el agua, obtengan el diploma de socorrista acuático. Un ejemplo de lo que se trata de transmitir estaría representado por la transformación de la prueba "300 metros de nado libre con ropa" con un tiempo disponible para su realización de ocho minutos, a la prueba "300 metros de nado libre" con el mismo tiempo disponible para su ejecución. Con independencia de la pertinencia de dicha prueba para la evaluación de socorristas acuáticos, es indiscutible que, si se dispone del mismo margen de tiempo, es más fácil superar la prueba cuando los 300 metros se nadan con un bañador, que cuando se nadan con un pantalón y una camiseta.

Como se ha dicho anteriormente, los resultados también muestran que las marcas de tiempo acreditadas por los socorristas acuáticos diplomados antes de la entrada en vigor de la Orden 1319/2006, son significativamente mejores que las acreditadas por aquellos que se diplomaron después de la entrada en vigor de dicha orden. La cuestión de fondo es que, en el contexto de la cadena de supervivencia, los segundos pueden marcar la diferencia entre el éxito y el fracaso (Austin \& Macintosh, 2013; Martínez y Hooper, 2014; Szpilman et al., 2014; Neumar et al., 2015). Además, se recuerda que cuando la víctima extraída del agua requiere un masaje cardíaco, la fatiga del rescatador influye negativamente sobre el resultado final de las maniobras de resucitación (Abelairas Gómez, Romo Pérez y Barcala Furelos, 2013). Esta evidencia induce a pensar que después de la fase de rescate acuático, aquellos socorristas que son más competentes en el agua, podrán iniciar las maniobras de resucitación 
con un menor nivel de fatiga, porque están mejor adaptados al ejercicio intenso en el agua. No menos importante es el hecho de que los socorristas asumen un gran riesgo al realizar rescates en el agua (Real Federación Española de Salvamento y Socorrismo, 2019) y aunque los medios y recursos actuales pueden disminuir el peligro, siempre existirá un mayor riesgo para aquellos rescatadores que no dominen adecuadamente el medio acuático.

Por último, indicar que no se han encontrado evidencias científicas que lo demuestren, pero todo hace pensar que, si al inicio de los cursos de formación de socorrista los aspirantes dispusieran de un adecuado nivel de competencia en el agua, se podría dedicar más tiempo a la enseñanza y a la práctica de las habilidades y competencias específicas del salvamento y socorrismo, en lugar de dedicar ese tiempo y esfuerzo, a la enseñanza y evaluación de competencias que pueden ser adquiridas en cursos de aprendizaje o perfeccionamiento de los estilos de natación y las habilidades acuáticas básicas. De ahí que, algunas entidades establezcan pruebas físicas de agua que deben ser superadas por el aspirante a socorrista, antes de iniciar el curso de formación (International Life Saving Federation, 2013).

Por todo lo anterior, se recomienda que las pruebas que se utilicen para evaluar a los aspirantes a socorrista sirvan para fomentar la mejora del nivel de competencia en el agua de este colectivo profesional y, por tanto, para incrementar la seguridad de los bañistas y del propio socorrista.

\section{CONCLUSIONES}

En respuesta al objetivo planteado en este estudio se concluye que, después de la aplicación de la norma de la Comunidad Autónoma de Madrid, se ha incrementado un $16 \%$ el porcentaje de aspirantes que obtiene el diploma de socorrista acuático, pero se ha disminuido significativamente $(p \leq 0,001)$ el nivel de competencia en el agua de estos profesionales. Por lo tanto, se recomienda que las entidades e instituciones que regulan la formación de socorristas acuáticos establezcan objetivos y criterios de evaluación que induzcan a la mejora del nivel de competencia en el agua de estos profesionales.

\section{REFERENCIAS BIBLIOGRÁFICAS}

Abelairas Gómez, C., Romo Pérez, V., \& Barcala Furelos, R. (2013). Efecto de la fatiga física del socorrista en los primeros cuatro minutos de la reanimación cardiopulmonar posrescate acuático. Emergencias, 25, 184190.

Austin, S., \& Macintosh, I. (2013). Management of drowning in children. Paediatrics and Child Health, 23(9), 397-401. https://doi.org/10.1016/i.paed.2013.05.018

Consejería de Sanidad y Consumo. (2006). Orden 1319/2006, de 27 de junio, de la Consejerìa de Sanidad y Consumo, por la que se establecen los criterios que permitan establecer los niveles de formación del personal que preste sus servicios como socorrista en piscinas, instalaciones 
acuáticas y medio natural de la Comunidad de Madrid. Boletín Oficial de la Comunidad de Madrid 14 de julio,(166), 53-82.

Decreto 80/1998, de 14 mayo de la Consejería Sanidad y Servicios Sociales por el que se regulan las Condiciones higiénico-sanitarias de las piscinas de uso colectivo (1998).

Cruz Roja. (2020). Socorrista acuático, 2020, from http://www.cruzroja.es/principal/web/formacion/informacioncurso?cod curso $=446503$

Ellis \& Associates. (2002). El profesional del rescate acuático. Barcelona: Paidotribo.

Federación Madrileña de Salvamento y Socorrismo. (2017). Curso de Formación de Socorrista en Playa y Medio Natural 2017, from http://www.fmss.es/ajax.php?accion=modalcomo-es-elcurso\&id curso $=374$

García Sanz, A., García Sanz, J. L., \& Díez Herrero, J. M. (2015). Técnicas de rescate y lesión medular en el medio acuático (Real Federación Española de Salvamento y Socorrismo ed.). España: Difusión Avances de Enfermería.

Harrell, A. (2001). Does supervision by lifeguards make a difference in rule violations? Effects of lifeguards. Psychological Reports, 89(2), 327-330. https://doi.org/10.2466/pr0.2001.89.2.327

International Life Saving Federation. (2000). International Life Saving Federation, Pool Lifeguard 2017, from http://www.ilsf.org/sites/ilsf.org/files/Certification/ILSCertificates/APP\%20 08\%20ILS\%20Lifeguard\%20Pool.pdf

International Life Saving Federation. (2007). Fitness Testing for Lifeguards, from http://www.ilsf.org/content/fitness-testing-lifeguards

International Life Saving Federation. (2013). International Life Saving Federation, beach lifeguard., 2017, from http://www.ilsf.org/sites/ilsf.org/files/Certification/ILSCertificates/APP\%20 10\%20ILS\%20Lifeguard\%20Beach.pdf

International Life Saving Federation. (2019). Competition Rule Book, Rules, Standards and Procedures for

Lifesaving World Championships and

ILS-Sanctioned Competitions. Leuven, Belgium: International Life Saving Federation,. Retrieved from https:/www.ilsf.org/wpcontent/uploads/2019/10/ILS-2019-Competition-Rulebook-Final-as-at-15October.pdf.

Martínez, F., \& Hooper, J. (2014). Drowning and inmersion injury. Anaesthesia \& Intensive Care Medicine, 15(9), 420-423. https://doi.org/10.1016/j.mpaic.2014.06.006

Ministerio de Trabajo e Inmigración. (2011). Real Decreto 711/2011, de 20 de mayo, por el que se establecen tres certificados de profesionalidad de la familia profesional Actividades físicas y deportivas que se incluyen en el Repertorio Nacional de certificados de profesionalidad. Boletín Oficial del Estado de 20 de mayo(711), 59404-59439.

Neumar, R. W. C., Shuster, M., Callaway, C. W., Gent, L. M., Atkins, D. L., Bhanji, F., . . . Hazinski, M. F. (2015). American Heart Association Guidelines 
Update for Cardiopulmonary Resuscitation and Emergency Cardiovascular Care. Supplement to Circulation, 132(8), S315-S367. https://doi.org/10.1161/CIR.0000000000000252

Palacios Aguilar, J. (2008). Socorrismo acuático profesional "formación para la prevención y la intervención de accidentes en el medio acuático. A Coruña SADEGA.

Pelletier, R., \& Gilchrist, J. (2011). Fatalities in swimming pools with lifeguards: USA 2000-2008. Injury Prevention, 17(4), 250-253. https://doi.org/10.1136/ip.2010.029751

Real Federación Española de Salvamento y Socorrismo. (2019). Muertes por ahogamiento en los espacios acuáticos españoles. Periodo 2015-2019

Sanz-Arribas, I. (2018). Influencia de la longitud del vaso sobre las pruebas de socorrismo acuático profesional. Revista Internacional de Medicina y Ciencias de la Actividad Física y el Deporte 18 (72), 783-795. https://doi.org/10.15366/rimcafd2018.72.012

Sanz Arribas, I. (2011). La coordinación de socorristas en piscinas con gran superficie de lámina de agua. Revista Internacional de Medicina y Ciencias de la Actividad Física y el Deporte, 11(44), 650-673.

Schwebel, D. C., Heater, J., Holder, E., \& Marciani, F. (2010). Lifeguards: A forgotten aspect of drowning prevention. Injure \& Violence, 2(1), 1-3. https://doi.org/10.5249/jivr.v2i1.32

Szpilman, D., \& Marcio, S. (2004). In-water resuscitation-is it worthwhile? Resuscitation, 63(1), 25-31. https://doi.org/10.1016/..resuscitation.2004.03.017

Szpilman, D., Webberemail, J., Quanemail, L., Bierensemail, J., MorizotLeiteemail, L., Langendorferemail, S. J., . . . Lofgrenemail, B. (2014). Creating a drowning chain of survival. Resuscitation, 85(9), 1149 - 1152. https://doi.org/10.1016/i.resuscitation.2014.05.034

The United States Lifesaving Association. (2016). Guidelines for open water lifeguard agency certification, from http://c.ymcdn.com/sites/www.usla.org/resource/resmgr/docs/usla certgu idelines.pdf

Número de citas totales / Total references: 27 (100\%).

Número de citas propias de la revista / Journal's own references: 2 $(7,41 \%)$.

Rev.int.med.cienc.act.fís.deporte - vol. 20 - número 80 - ISSN: 1577-0354 